\title{
Apparatus for Characterizing Gas-Phase Chemical Precursor Delivery for Thin Film Deposition Processes
}

\author{
James E. Maslar, William A. Kimes, and Brent A. Sperling \\ National Institute of Standards and Technology, \\ Gaithersburg, MD 20899, USA \\ james.maslar@nist.gov \\ william.kimes@nist.gov \\ brent.sperling@nist.gov
}

\begin{abstract}
Thin film vapor deposition processes, e.g., chemical vapor deposition, are widely used in high-volume manufacturing of electronic and optoelectronic devices. Ensuring desired film properties and maximizing process yields require control of the chemical precursor flux to the deposition surface. However, achieving the desired control can be difficult due to numerous factors, including delivery system design, ampoule configuration, and precursor properties. This report describes an apparatus designed to investigate such factors. The apparatus simulates a single precursor delivery line, e.g., in a chemical vapor deposition tool, with flow control, pressure monitoring, and a precursor-containing ampoule. It also incorporates an optical flow cell downstream of the ampoule to permit optical measurements of precursor density in the gas stream. From such measurements, the precursor flow rate can be determined, and, for selected conditions, the precursor partial pressure in the headspace can be estimated. These capabilities permit this apparatus to be used for investigating a variety of factors that affect delivery processes. The methods of determining the pressure to (1) calculate the precursor flow rate and (2) estimate the headspace pressure are discussed, as are some of the errors associated with these methods. While this apparatus can be used under a variety of conditions and configurations relevant to deposition processes, the emphasis here is on low-volatility precursors that are delivered at total pressures less than about $13 \mathrm{kPa}$ downstream of the ampoule. An important goal of this work is to provide data that could facilitate both deposition process optimization and ampoule design refinement.
\end{abstract}

Keywords: ALD; atomic layer deposition; CVD; chemical vapor deposition; precursor delivery; thin film deposition.

Accepted: February 12, 2019

Published: March 26, 2019

https://doi.org/10.6028/jres.124.005

\section{Introduction}

Thin film vapor deposition processes, e.g., chemical vapor deposition (CVD) and atomic layer deposition (ALD), are widely used in high-volume manufacturing of electronic and optoelectronic devices. Ensuring desired film properties and maximizing process yields require control of the chemical precursor flux to the deposition surface. For precursors that are gases at the delivery conditions, achieving such control using mass flow controllers (MFCs) and pressure transducers is relatively straightforward. However, for precursors that are low-volatility liquids or solids, e.g., many organometallic compounds, achieving the desired control is more difficult. Such precursors are often delivered using a carrier gas that is passed through either a bubbler, i.e., a vessel with a dip tube, or a vapor draw ampoule, i.e., a vessel with no dip tube in which the gas-in and gas-out ports open directly into the ampoule headspace. A lack of adequate control can result in an irreproducible amount of entrained precursor, and the negative impacts of such variations on film and device properties have been widely reported [1-10]. Several factors can contribute to an inadequate level of control, including the design of the gas manifold $[5,7,8,11]$, the design of the ampoule temperature-control system [12], the gas flow path in the ampoule [13-15], evaporative/sublimative cooling of the precursor in the ampoule [16, 17], and the changing surface area of solid precursors in the ampoule 
(due to sublimation and subsequent recrystallization) $[14,15,18]$. These factors can be grouped into three categories: (1) delivery apparatus; (2) ampoule design; and (3) precursor properties.

The desire to better understand the impact of these factors on precursor delivery has motivated efforts to develop methods to investigate gas-phase precursor delivery processes. In situ optical techniques have been used to characterize different aspects of precursor delivery processes, since these techniques can be robust, rapid, and minimally perturbing to the process under investigation $[19,20]$. The type of information that can be obtained with an optical technique, and ultimately the applicability of the results, depends on the complementary data that are available. For a precursor delivered with a carrier gas, one can identify three cases: (1) a calibrated optical technique with no supporting data (i.e., the optical response can be converted to precursor density); (2) a calibrated optical technique with both the carrier gas flow rate and total pressure at the optical flow cell being known; and (3) a calibrated optical technique with the carrier gas flow rate, pressure at the optical flow cell, and pressure in the ampoule headspace being known. These three situations will be discussed subsequently.

In the first case, time-dependent measurement of precursor density (partial pressure) can provide insight into precursor delivery stability and process parameters affecting the stability. However, the precursor partial pressure at any location in the delivery system depends strongly on the specifics of the delivery system design, e.g., the existence of a drop between the ampoule headspace and the measurement location [21-25]. Hence, in this case, it is difficult to distinguish delivery apparatus factors from ampoule design and precursor property factors, potentially limiting the applicability of results in this case.

In the second case, knowledge of the precursor partial pressure as well as knowledge of the carrier gas flow rate and the total pressure at the optical cell (e.g., obtained from an MFC and a pressure transducer reading, respectively) permits the determination of the precursor flow rate using the "bubbler equation" [12, 16, 21, 22, 24, 26, 27]:

$$
F_{\mathrm{pr}}=F_{\mathrm{cr}} \frac{P_{\mathrm{pr}}^{x}}{P_{\mathrm{cr}}^{x}}
$$

where $F_{\mathrm{pr}}$ and $F_{\mathrm{cr}}$ are the precursor and carrier gas flow rate, respectively, and $P_{\mathrm{pr}}^{x}$ and $P_{\mathrm{cr}}^{x}$ are the precursor and carrier gas partial pressure, respectively, at a location " $x$ " in the flow system, and $P_{\mathrm{total}}^{x}=P_{\mathrm{pr}}^{x}+P_{\mathrm{cr}}^{x}$, where $P_{\mathrm{total}}^{x}$ is the total pressure at $x$. In this case, $x$ corresponds to the flow cell location. For conditions described by Eq. (1), the respective flow rates and the $P_{\mathrm{pr}}^{x} / P_{\mathrm{cr}}^{x}$ ratio are constant throughout the system (assuming ideal gas behavior). Hence, the flow properties of the delivery apparatus are well characterized, making it relatively straightforward to identify and compare apparatus-related properties across test systems. (This differs from the previous case, in which only the precursor partial pressure is measured). Furthermore, $F_{\mathrm{pr}}$ can be used to calculate a source efficiency term, which can help to quantify system nonideality [28] (although not necessarily aid in the identification of sources of any nonideality).

Even with the complementary data of the second case, obtaining further insight into how ampoule design or precursor properties affect precursor delivery can be difficult. Such insight generally requires knowledge of the precursor partial pressure in the ampoule headspace. Ideally, the precursor partial pressure could be estimated based on a knowledge of the precursor vapor pressure at the ampoule temperature (which is how the bubbler equation is typically employed to estimate precursor flow rate) [12, 16, 22, 24, 26, 29]. Unfortunately, this ideal situation is often not realized $[12,15,16,27,30]$. Alternatively, the precursor partial pressure in the headspace could be estimated from a knowledge of the carrier gas partial pressure in the headspace using the bubbler equation (assuming that the precursor and carrier gas flow rates are known). (This approach also assumes that no precursor partial pressure gradient exists in the headspace, an assumption that may be invalid for some ampoule configurations and flow conditions.) The simplest approach to obtain headspace pressure is to install a pressure transducer on the ampoule. Unfortunately, it can be undesirable to do so on an ampoule containing a reactive precursor. Another approach is to relate a pressure measurement made outside the ampoule to the pressure in the headspace. Determining this relationship can be difficult: The difficulty arises because at least one valve typically separates the headspace and the transducer, and the pressure drop as a function of flow through any such valves is not necessarily well characterized. However, a pressure transducer will generally already be installed on the delivery line, a 
situation which would facilitate this approach. Despite these difficulties, if an estimate of the precursor partial pressure in the ampoule headspace can be obtained, then the effects of ampoule design and precursor properties could be more thoroughly investigated, thereby further expanding the applicability of results obtained in this case.

The objective of this report is to describe an apparatus that, when coupled with an in situ optical technique, can be used to characterize gas-phase precursor delivery processes, especially for CVD and ALD applications (e.g., see Ref. [31]). The flow system was designed to approximate a single precursor delivery line with an optical flow cell installed downstream of a precursor-containing ampoule to permit optical measurements in the gas stream. Pressure is recorded upstream of the ampoule and downstream of the flow cell. No pressure transducer is placed between the ampoule and flow cell, because doing so could introduce perturbations to time-dependent measurements due to the presence of the tube connecting the transducer to the delivery line. (This "dead volume" would be poorly swept by the carrier gas flow, resulting in relatively large residence times in the connecting tube.) The carrier gas pressure at the flow cell can be calculated from the downstream pressure transducer data, enabling the calculation of the corresponding precursor flow rate. Although no pressure transducer is located near the ampoule headspace, the pressure near the ampoule outlet can be calculated from the downstream transducer data, and these data in combination with the upstream transducer data can be used to identify the maximum pressure drop across the ampoule valves. Furthermore, for selected valve configurations, the headspace pressure can be estimated from these data, when the valve flow properties are characterized. Hence, this apparatus was designed with the capability to investigate delivery apparatus, ampoule design, and precursor property factors that affect delivery processes. The errors associated with determination of the pressure at the flow cell, ampoule outlet, and ampoule headspace are discussed. While, in principle, any precursors can be examined with this system, the emphasis of this report will be on low-volatility precursors that are delivered on a system with pressures less than $13 \mathrm{kPa}$ downstream of the ampoule. Such precursors can be particularly difficult to reproducibly deliver but are being increasingly utilized in semiconductor fabrication processes. An additional emphasis of this report will be on commercial $1.5 \mathrm{~L}$ ampoules (with a maximum fill of $1.2 \mathrm{~L}$ ), particularly those incorporating a five-valve cluster. Such containers are commonly used to supply low-volatility chemical precursors for complementary metal-oxide-semiconductor (CMOS)-based manufacturing processes. The data obtained from investigations based on the apparatus described here should facilitate both process optimization and development of improved ampoule designs.

\section{Design of the Apparatus}

Figure 1 shows a schematic of the apparatus. A carrier gas flow is controlled by an MFC with a full-scale range of $2 \mathrm{~L} / \mathrm{min}$ at standard temperature and pressure (STP), defined as $0{ }^{\circ} \mathrm{C}$ and $101.33 \mathrm{kPa}$. In subsequent discussions, gas flow rates are referenced to STP unless otherwise noted. Gas is supplied to the MFC from a compressed gas cylinder using a high-purity regulator followed by a point-of-use gas purifier (not shown). Pressure in the apparatus is measured at a minimum of two locations using capacitance diaphragm gauges (CDGs): CDG1 upstream of the valve cluster and CDG2 downstream of the flow cell. To measure the pressure at other locations under selected conditions, an additional CDG was temporarily installed either on the fill port of the ampoule or between the two elbows downstream of the ampoule outlet. CDG1, CDG2, and any additional CDG had full-scale ranges of $133.3 \mathrm{kPa}$ (1000 Torr), $13.3 \mathrm{kPa}$ (100 Torr), and $13.3 \mathrm{kPa}$, respectively, and all CDGs were temperature-controlled at $100^{\circ} \mathrm{C}$. The system is depicted with a $1.5 \mathrm{~L}$, five-valve cluster ampoule (the ampoule configuration will be discussed in more detail subsequently). Optical access to the delivery line was achieved via the installation of an inline optical flow cell (FC) located $32 \mathrm{~cm}$ upstream of CDG2 (the FC design will be discussed in more detail subsequently). The conductance of the flow system can be controlled using the manual throttle (butterfly) valve (TV). This valve has a clear aperture of $\sim 19 \mathrm{~mm}$, and the valve stem was coupled to a manual rotation stage for improved control of the valve disc position, i.e., the conductance. The system can be isolated from the vacuum pump using the high-conductance bellows isolation valve (IV). Anticipating a subsequent discussion, $P_{\mathrm{c}}^{\text {OUT }}$ refers to the calculated pressure at a location $6 \mathrm{~cm}$ from the ampoule outlet elbow (or $102 \mathrm{~cm}$ upstream of CDG2). This pressure value can be used to estimate (1) the maximum pressure drop across the valve cluster (by subtracting the CDG1 


\section{Journal of Research of the National Institute of Standards and Technology}

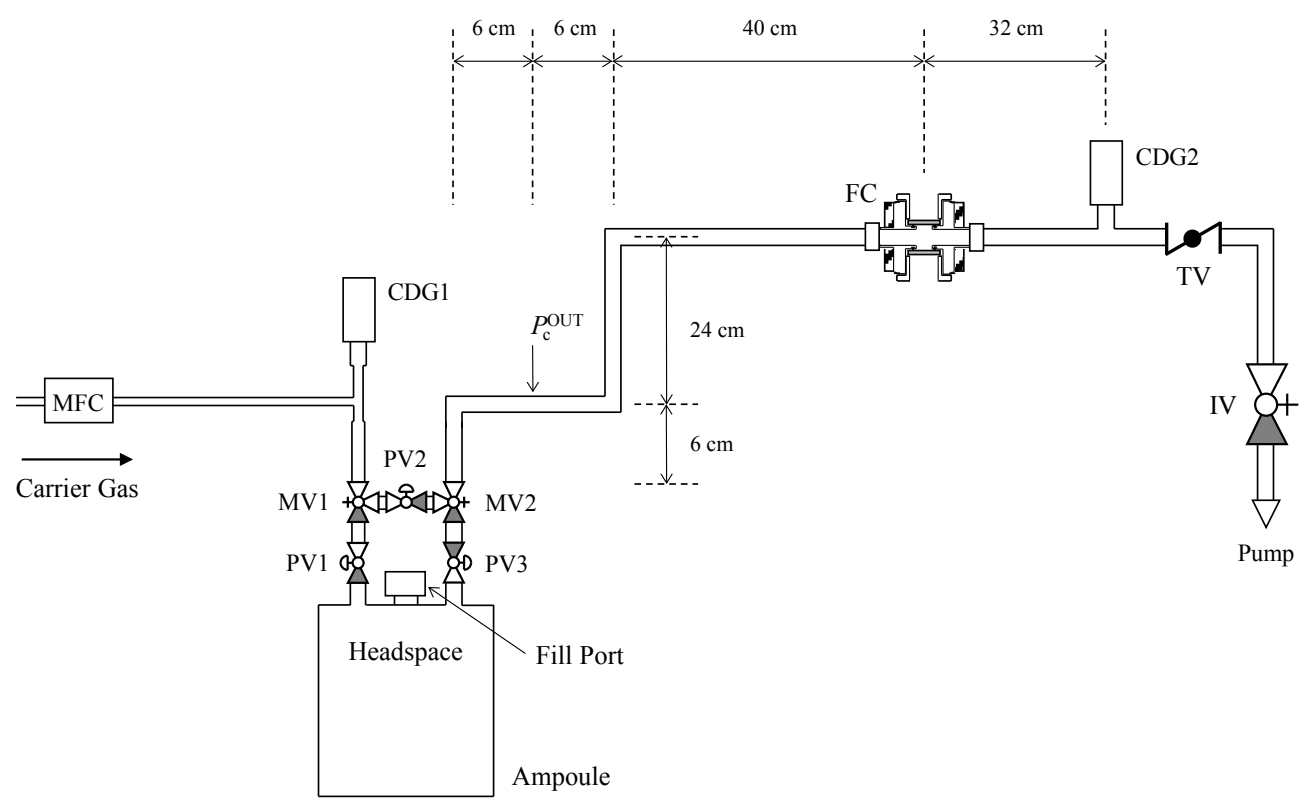

Fig. 1. Schematic diagram of the apparatus. MFC, mass flow controller; CDG1 and CDG2, capacitance diaphragm gauges; PV1, PV2, and PV3, pneumatically actuated diaphragm valves; MV1 and MV2, manual diaphragm valves; FC, optical flow cell; TV, throttle valve; IV, isolation valve; $P_{\mathrm{c}}^{\text {OUT }}$, the pressure calculated at $6 \mathrm{~cm}$ downstream of the elbow at the ampoule outlet. The drawing is not to scale.

pressure) and (2) the headspace pressure (when the flow characteristics of the injection valves are known). While locating the position of $P_{\mathrm{c}}^{\mathrm{OUT}}$ closer to the ampoule outlet could improve these estimates, a position equidistant from the two elbows was selected because it was assumed that flow perturbations due to the elbow geometry would be minimized at this location.

All surfaces upstream of IV that may be exposed to the precursor were insulated and could be heated (to avoid precursor condensation). Ampoules tested with this apparatus were typically encased in an aluminum jacket, and the sides and bottom were actively heated with flexible strip heaters while the top plate was passively heated. The valves in the valve cluster were individually encased in aluminum jackets and heated with heating tape, or the whole valve cluster was covered with a commercially available heating jacket designed specifically for the valve cluster. The optical flow cell was encased in an aluminum jacket and heated with cartridge heaters. All other lines were heated using heating tapes. Mineral-insulated, metal-sheathed type $\mathrm{K}$ thermocouples were used to measure the temperature for active temperature control. The estimated temperature accuracy was $\pm 2.2{ }^{\circ} \mathrm{C}$ at $250{ }^{\circ} \mathrm{C}$ with $k=2$ (where $k$ is a numerical factor corresponding to the desired confidence level), based on manufacturer's specifications. All lines were composed of commercially available, electropolished stainless-steel tubing, with $0.64 \mathrm{~cm}$ ( $0.25 \mathrm{in})$ outside diameter (OD) tube being used for the section upstream of CDG1 and $1.3 \mathrm{~cm}(0.5 \mathrm{in})$ OD tube being used for the section between the ampoule and TV. A perfluoropolyether lubricant-filled rotary vane vacuum pump with a specified pumping speed of $26 \mathrm{~m}^{3} \cdot \mathrm{h}^{-1}$ and an ultimate base pressure of $<0.2 \mathrm{~Pa}$ was utilized.

The ampoule was configured with a five-valve cluster that included three pneumatic 2-port valves (PV1, PV2, and PV3) and two manual 3-port valves (MV1 and MV2). The manual 3-port valves were configured in a "T," in which one side of the arm of the " $\mathrm{T}$ " was valved and flow was unimpeded through the stem of the "T," and the other side of the arm was nonvalved. The two manual valves were used to isolate the ampoule from the flow system when measurements were not being performed. The focus of this report will be on this type of an ampoule, but ampoules with different valve configurations can be readily examined with appropriate connections to the delivery lines. For example, in the case of an ampoule configured only with two manual isolation valves (one each on the inlet and outlet), a three-valve stick consisting of two pneumatically actuated 3-port valves on each side of one pneumatically actuated 2-port valve could be used to control precursor injection into the flow system. (In this case, the 3-port 
valves could be configured with the stem of the " $\mathrm{T}$ " being valved and flow being unimpeded through the arm of the "T.") Commercial ampoules are fabricated with a fill port that is sealed with a metal gasket-sealed cap.

The optical flow cell was designed to minimally perturb flow by approximating flow through a tube. The body consisted of a stainless-steel block with a $10.2 \mathrm{~mm}$ diameter bore (nominally matching the internal diameter of the $1.3 \mathrm{~cm}$ [0.5 in] OD delivery line tube), to which commercially available metal gasket face seal glands were welded. The block form factor was selected to provide mounting surfaces for windows and window clamps. The body accepted two $25.4 \mathrm{~mm}$ diameter windows on opposite sides of the block. The window-to-body seals were made with fluoropolymer elastomer O-rings. A commonly employed window was an uncoated, $5.1 \mathrm{~mm}$ thick $\mathrm{ZnSe} 0.5$ degree wedge (wedged to reduce étalon effects). The optical path length from window surface to window surface inside the cell was $15.2 \mathrm{~mm}$. The optical path in the laboratory ambient environment was typically enclosed with lens tubes and purged with nitrogen to minimize optical intensity fluctuations on the detector, which can occur when the flow cell is heated [32]. The direction of optical radiation propagation was perpendicular to the direction of gas flow.

The specified MFC settling time was less than $750 \mathrm{~ms}$, and the typical accuracy was $\pm 1 \%$ of the set point for flow rates of $500 \mathrm{~mL} / \mathrm{min}$ and $750 \mathrm{~mL} / \mathrm{min}$ (STP) and $\pm 0.2 \%$ of full scale for $250 \mathrm{~mL} / \mathrm{min}$. The specified CDG response time was $30 \mathrm{~ms}$, and the typical accuracy was $\pm 0.2 \%$ of the gauge reading.

\section{Operating Characteristics of the Apparatus}

Flow in the system is initiated in the purge configuration (with no flow through the ampoule) by opening the pneumatic valve PV2, with PV1 and PV3 being closed (MV1 and MV2 are open for all measurements), setting the MFC to the desired flow rate, and adjusting TV to obtain the desired pressure in the flow cell, as measured on CDG2. The injection configuration (the configuration used to inject precursor) involves directing carrier gas through the ampoule, and in this configuration, PV2 is closed, with PV1 and PV3 being open. All measurements described here were performed in either of these two configurations, although only carrier gas was injected for these studies. For all measurements described in this report, ultrahigh-purity grade argon was used as the carrier gas. To obtain the pressure values reported in this work, CDG readings were recorded at $200 \mathrm{~Hz}$ for $240 \mathrm{~s}$ at each set of conditions, and the average of the final $30 \mathrm{~s}$ of data was taken as the pressure value.

The pressure characteristics of the apparatus are illustrated in Fig. 2, which shows the pressure at different locations in the (a) bypass configuration and (b) injection configuration at a $1.00 \mathrm{~L} / \mathrm{min}$ argon flow rate for seven TV settings (denoted TV-1 to TV-7) and set point temperatures of $75^{\circ} \mathrm{C}, 80^{\circ} \mathrm{C}$, and $90^{\circ} \mathrm{C}$ for the ampoule, valve cluster, and lines, respectively. Pressures were measured (solid circles) at CDG1 ( $\left.P_{\mathrm{m}}^{\mathrm{CDG} 1}\right)$, the ampoule headspace $\left(P_{\mathrm{m}}^{\mathrm{HS}}\right)$, and CDG2 $\left(P_{\mathrm{m}}^{\mathrm{CDG} 2}\right)$, while pressures were calculated (open circles) at the location corresponding to $P_{\mathrm{c}}^{\text {OUT }}$ and at the flow cell $\left(P_{\mathrm{c}}^{\mathrm{FC}}\right)$. The method of calculation will be discussed subsequently. The flow was progressively restricted by adjusting the TV to seven positions, denoted TV-1 (for which the throttle valve was fully open) to TV-7 (for which TV was adjusted to provide $\sim 6 \mathrm{kPa}$ on CDG2). In Fig. $2, P_{\mathrm{m}}^{\mathrm{CDG} 1}$ and $P_{\mathrm{m}}^{\mathrm{CDG} 2}$ values range from $\sim 2 \mathrm{kPa}$ to $\sim 7 \mathrm{kPa}$ and from $\sim 0.45 \mathrm{kPa}$ to $\sim 6 \mathrm{kPa}$, respectively, with the magnitude of the pressure drop between CDG1 and CDG2 decreasing with decreasing throttle conductance. In the bypass configuration, the largest pressure drop is observed between $P_{\mathrm{m}}^{\mathrm{CDG1}}$ and $P_{\mathrm{c}}^{\mathrm{OUT}}$ (i.e., across valve PV2) for a given TV setting. In the injection configuration, the largest pressure drop is observed between $P_{\mathrm{m}}^{\mathrm{HS}}$ and $P_{\mathrm{c}}^{\mathrm{OUT}}$ (i.e., across the exit of the ampoule and the two valves downstream of the ampoule) for a given TV setting. Hence, the pressure drop downstream of the ampoule is larger than that upstream in the injection configuration. The $P_{\mathrm{m}}^{\mathrm{CDG} 2}$ values observed in the bypass and injection configurations are nominally identical for a given TV setting. This is consistent with a relatively high conductance in the $1.3 \mathrm{~cm}$ ( 0.5 in) OD delivery line. The characteristics illustrated in Fig. 2 are typical for all flow rates, although the pressure values obviously are different. Increasing the flow rate to $1.50 \mathrm{~L} / \mathrm{min}$ resulted in corresponding pressure values that were $40 \%$ to $45 \%$ higher, while decreasing the flow rate to $0.25 \mathrm{~L} / \mathrm{min}$ resulted in corresponding pressure values that were $55 \%$ to $65 \%$ lower.

Gas flow at the conditions shown in Fig. 2 can be characterized by the Reynolds number (Re), Knudsen number $(\mathrm{Kn})$, and Mach number (Ma). $\mathrm{Kn}$ is calculated from [33]: 


$$
\mathrm{Kn}=\frac{\lambda}{d}=\frac{k T_{\mathrm{act}}}{d\left(2^{1 / 2} \pi d_{\mathrm{Ar}}^{2} P_{\mathrm{act}}\right)},
$$

where $\lambda$ is the mean free path $(\mathrm{m}), k$ is the Boltzmann constant $(\mathrm{J} / \mathrm{K}), T_{\text {act }}$ is the actual absolute temperature $(\mathrm{K}), d$ is the tube inside diameter $(\mathrm{m}), d_{\mathrm{Ar}}$ is the effective diameter of an argon atom $(\mathrm{m})$, and $P_{\text {act }}$ is the actual pressure $(\mathrm{Pa})$. The $d_{\mathrm{Ar}}$ value is estimated from the reported argon viscosity, $\eta(\mathrm{Pa} \cdot \mathrm{s})$ [34]:

$$
d_{\mathrm{Ar}}^{2}=\frac{\sqrt{k T_{\mathrm{act}} m}}{\pi^{3 / 2} \eta}
$$

where $m$ is mass of an argon atom $(\mathrm{kg})$. Re is calculated from [33]:

$$
\mathrm{Re}=\frac{4 m Q}{\pi k T_{\mathrm{act}} \eta d},
$$

where $Q$ is the throughput $\left(\mathrm{Pa} \cdot \mathrm{m}^{3} / \mathrm{s}\right)$, which is calculated from

$$
Q=F_{\mathrm{STP}} \frac{T_{\mathrm{act}} P_{\mathrm{STP}}}{T_{\mathrm{STP}}},
$$

where $F_{\mathrm{STP}}, P_{\mathrm{STP}}$, and $T_{\mathrm{STP}}$ are the carrier gas flow rate, pressure, and absolute temperature, respectively, at STP. $\mathrm{Ma}$ is calculated from [33]:

$$
\mathrm{Ma}=\frac{U}{U_{\text {sound }}}=\frac{4 Q}{\pi d^{2} P_{\text {act }} U_{\text {sound }}}
$$

where $U_{\text {sound }}$ is the sound velocity $(\mathrm{m} / \mathrm{s})$ and was obtained from

$$
U_{\text {sound }}=v\left(\frac{\pi \gamma}{8}\right)^{1 / 2}
$$

where $\gamma$ is the ratio of specific heats of argon, $\gamma \approx 1.667$ for a monatomic gas, and $v$ is the average particle velocity given by

$$
v=\left(\frac{8 k T_{\mathrm{act}}}{\pi m}\right)^{1 / 2} .
$$

In addition, flow through an orifice is identified as choked flow if the following condition is valid [33]:

$$
\frac{P_{\mathrm{c}}^{\mathrm{OUT}}}{P_{\mathrm{m}}^{\mathrm{CDG} 1}} \leq\left(\frac{2}{\gamma+1}\right)^{\gamma / \gamma-1}=0.487
$$



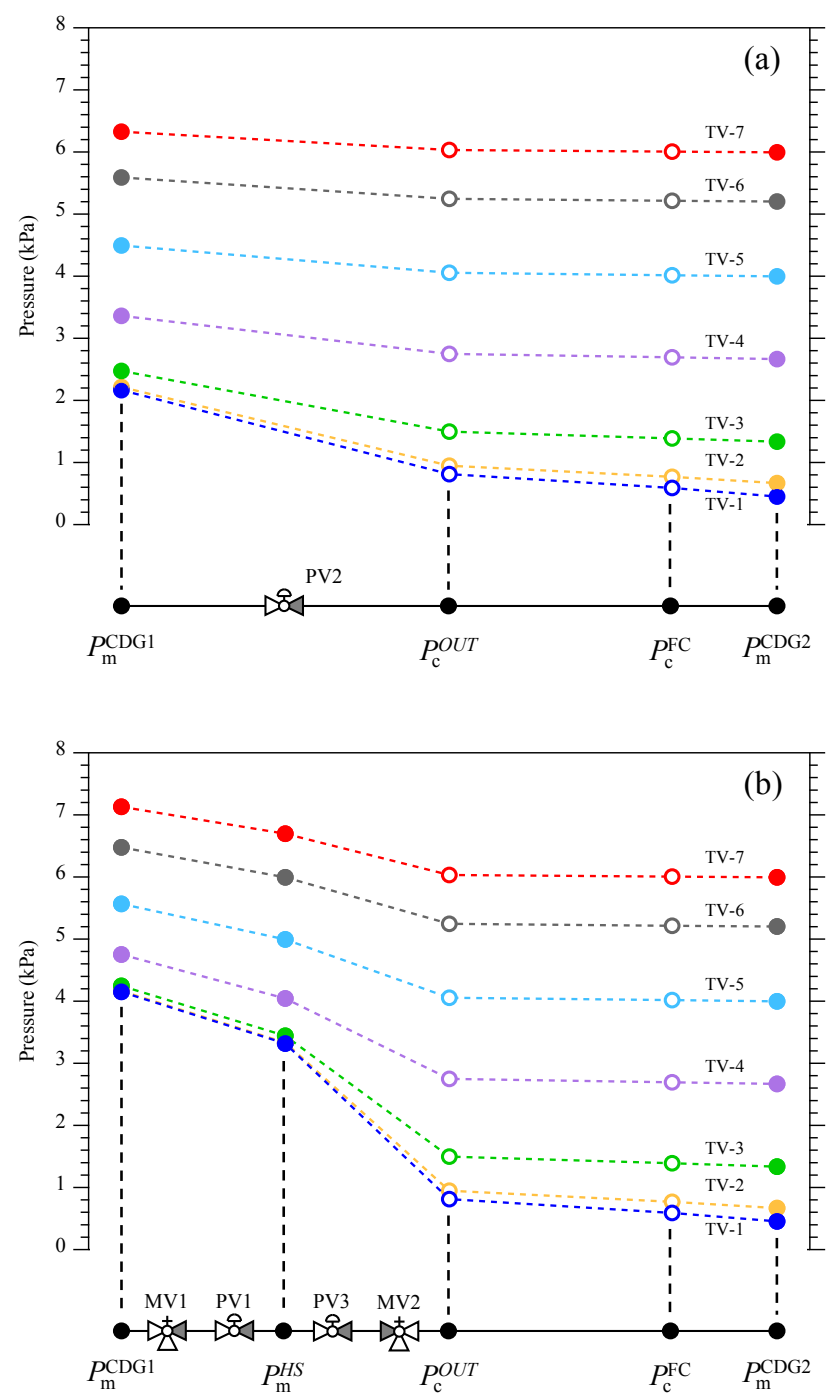

Fig. 2. The pressure at different locations in this apparatus in the (a) bypass configuration and (b) injection configuration for seven TV settings (denoted TV-1 to TV-7) at a $1.00 \mathrm{~L} / \mathrm{min}$ argon flow rate. Pressures were measured (solid circles) at CDG1 ( $\left.P_{\mathrm{m}}^{\mathrm{CDG} 1}\right)$, the ampoule headspace $\left(P_{\mathrm{m}}^{\mathrm{HS}}\right)$, and CDG2 $\left(P_{\mathrm{m}}^{\mathrm{CDG} 2}\right)$, and pressures were calculated (open circles) at the location corresponding to $P_{\mathrm{c}}^{\text {OUT }}$ and at the flow cell $\left(P_{\mathrm{c}}^{\mathrm{FC}}\right)$.

For a location near CDG2 and the conditions shown in Fig. 2, $\mathrm{Kn}<0.002, \mathrm{Re}<300$, and $\mathrm{Ma}<0.2$. Hence, carrier gas flow in the vicinity of CDG2 is in the continuous-flow regime $(\mathrm{Kn}<0.01)$ and is laminar $(\mathrm{Re}<1200)$ and incompressible $(\mathrm{Ma}<0.3)$. In fact, for a temperature of $100{ }^{\circ} \mathrm{C}$ and flow in a $1.3 \mathrm{~cm}(0.5 \mathrm{in}) \mathrm{OD}$ tube, continuum flow should be observed for pressures greater than $\sim 93 \mathrm{~Pa}(\sim 0.7$ Torr), which corresponds to the majority of conditions accessible with this apparatus. Transition flow $(1>\mathrm{Kn}>0.01)$ may be observed for lower flow rates at high conductance settings, at which lower pressures are observed. Furthermore, $\operatorname{Re}<300$ for flow rates up to $2.0 \mathrm{~L} / \mathrm{min}$ at $100{ }^{\circ} \mathrm{C}$ (i.e., laminar viscous flow should develop downstream of the ampoule nominally for all conditions accessible with this apparatus). At the valve cluster, the $P_{\mathrm{c}}^{\mathrm{OUT}} / P_{\mathrm{m}}^{\mathrm{CDG} 1}$ values for TV-1 and TV-2 are 0.375 and 0.428 , respectively, in the bypass configuration. Hence, flow is choked through PV2 for these conditions, which also correspond to higher Ma values. 


\section{Calculating the Pressure in the Delivery Line}

Knowledge of the pressure at the flow cell is necessary to calculate precursor flow rate using Eq. (1), and, anticipating a subsequent discussion, knowledge of the pressure near the ampoule outlet is useful to estimate the ampoule headspace pressure. The pressure in a long straight tube can be calculated by adapting the Hagen-Poiseuille equation for liquids to an ideal gas [33]:

$$
Q=\frac{\pi d^{4}}{128 \eta l} \frac{P^{\mathrm{up}}+P^{\mathrm{down}}}{2}\left(P^{\mathrm{up}}-P^{\mathrm{down}}\right),
$$

where $P^{\text {up }}$ and $P^{\text {down }}$ are the upstream and downstream pressure $(\mathrm{Pa})$, respectively, and $l$ is the distance $(\mathrm{m})$ between $P^{u p}$ and $P^{\text {down }}$. The Hagen-Poiseuille equation assumes (1) an ideal gas where (2) flow is laminar and (3) fully developed with (4) zero wall velocity. Assumptions (1), (2), and (4) are generally valid, since carrier gas flow is in the continuum-flow regime and is laminar and incompressible for typical conditions employed in this work, particularly near CDG2. Concerning assumption (3), flow from a large volume into a tube is considered to be fully developed for a distance, $l_{\mathrm{e}}(\mathrm{m})$, from the tube entrance that is given by [33]:

$$
l_{\mathrm{e}}=0.0568 d \mathrm{Re}
$$

For the conditions employed in this investigation, $l_{\mathrm{e}}$ values range from $\sim 2 \mathrm{~cm}$ to $\sim 13 \mathrm{~cm}$ at $0.2 \mathrm{~L} / \mathrm{min}$ to $1.5 \mathrm{~L} / \mathrm{min}$, respectively. Hence, flow should be fully developed well before the flow cell. The validity of this equation for calculating pressure in this apparatus was evaluated by temporarily installing a third CDG downstream of the ampoule outlet and comparing the measured and calculated pressures at this location, $P_{\mathrm{m}}^{\mathrm{H}-\mathrm{P}}$ and $P_{\mathrm{c}}^{\mathrm{H}-\mathrm{P}}$, respectively. This CDG was installed on the stem of an inverted " $T$ " that was inserted into the flow system equidistant from the two elbows downstream of the ampoule outlet. The "T" was constructed of $1.3 \mathrm{~cm}(0.5 \mathrm{in})$ OD tube. The base of the " $T$ " was $12 \mathrm{~cm}$ in length, and, hence, the position of this third CDG was $12 \mathrm{~cm}$ downstream of the elbow at the ampoule outlet and $108 \mathrm{~cm}$ upstream of CDG2. The value of $P_{\mathrm{m}}^{\mathrm{H}-\mathrm{P}}$ was obtained from $P_{\mathrm{m}}^{\mathrm{CDG} 3}-P_{\mathrm{m}}^{\mathrm{CDG} 2}$. The value of $P_{\mathrm{c}}^{\mathrm{H}-\mathrm{P}}$ was calculated using $P_{\mathrm{m}}^{\mathrm{CDG} 2}$ and rearranging Eq. (10) as follows,

$$
P_{\mathrm{c}}^{\mathrm{H}-\mathrm{P}}=\left[\left(\frac{256 Q \eta l}{\pi d^{4}}\right)+\left(P_{\mathrm{m}}^{\mathrm{CDG} 2}\right)^{2}\right]^{\frac{1}{2}} .
$$

Figure 3 shows (a) a comparison of the calculated and measured $P^{\mathrm{H}-\mathrm{P}}$ values and (b) the corresponding relative error of the $P_{\mathrm{c}}^{\mathrm{H}-\mathrm{P}}$ value for different carrier gas flow rates at a delivery line set point temperature of $63{ }^{\circ} \mathrm{C}$. The relative error is less than $\pm 3 \%$ at all pressures, with most values above $\sim 2.5 \mathrm{kPa}$ being between $0.2 \%$ and $0 \%$. The lower pressure data points at each flow rate tend to exhibit the larger relative errors. For the lowest pressure data point at each flow rate, flow is choked through PV2. Presumably, choked flow through the valve results in a greater distance for flow to be fully developed than the value estimated from Eq. (11), and this effect contributes to the larger relative errors at these conditions. Whatever the underlying reason(s) for the difference between the calculated and measured pressures, the differences are small, particularly when flow through PV2 is not choked. Furthermore, when calculating the pressure at the flow cell, the distance between the flow cell and CDG2 is significantly less than that between the two CDGs, and, hence, the corresponding error in the calculated pressure will be less. 

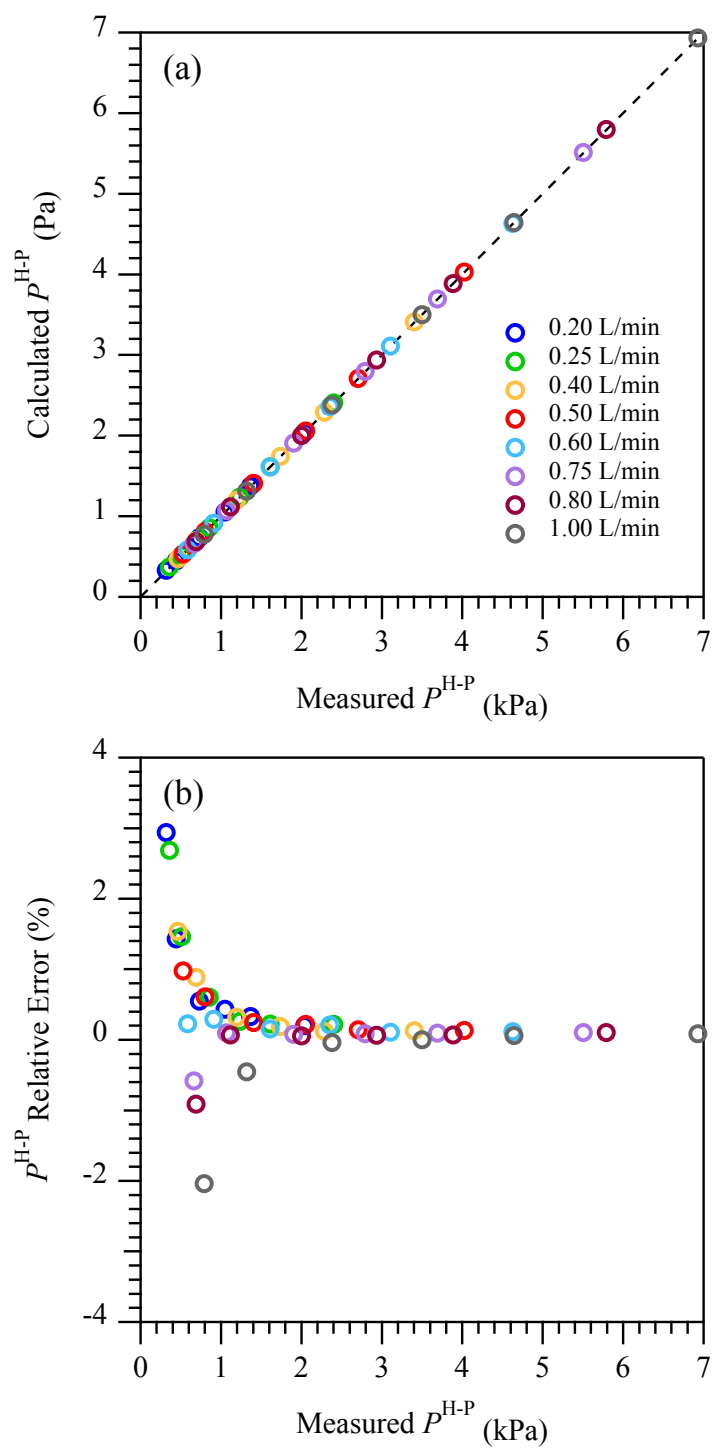

Fig. 3. (a) Calculated and measured $P^{\mathrm{H}-\mathrm{P}}$ values and (b) corresponding relative error at different carrier gas flow rates.

\section{Estimating the Pressure in the Ampoule Headspace}

An understanding of the precursor partial pressure in the ampoule headspace can provide insight into processes such as evaporative/sublimative cooling and mass transport in the ampoule. Although this value may be difficult to obtain directly, if one can estimate the carrier gas partial pressure in the headspace based on a pressure measurement outside the ampoule, then Eq. (1) can be used to calculate the precursor partial pressure in the headspace, assuming that the precursor and carrier gas flow rates are known. In the case of a small pressure drop across the valve cluster, the headspace pressure can be estimated based on the CDG1 reading and the $P_{\mathrm{c}}^{\text {OUT }}$ calculation. However, in the case of a large pressure drop across the valve cluster, a better estimate of the headspace pressure may be desirable. An alternative method is described subsequently. 


\section{Journal of Research of the National Institute of Standards and Technology}

The pressure in the headspace, $P^{\mathrm{HS}}$, is related to $P_{\mathrm{m}}^{\mathrm{CDG} 2}$ according to

$$
P^{\mathrm{HS}}=\Delta P^{\text {exit }}+\Delta P^{\mathrm{PV} 3}+\Delta P^{\mathrm{MV} 2}+\Delta P^{\mathrm{line}}+P_{\mathrm{m}}^{\mathrm{cDG} 2}=\Delta P^{\text {exit }}+\Delta P^{\mathrm{PV} 3}+\Delta P^{\mathrm{MV} 2}+P_{\mathrm{c}}^{\mathrm{OUT}},
$$

where $\Delta P^{\text {exit }}$ is the pressure drop at the ampoule exit, $\Delta P^{\mathrm{PV} 3}$ and $\Delta P^{\mathrm{MV} 2}$ are the pressure drop across valve PV3 and MV2, respectively, and $\Delta P^{\text {line }}$ is the pressure drop in the delivery line between CDG2 and the position corresponding to $P_{\mathrm{c}}^{\text {OUT }}$. Calculations of $P_{\mathrm{c}}^{\text {OUT }}$ are analogous to the calculations of $P_{\mathrm{c}}^{\mathrm{H}-\mathrm{P}}$ described in the previous section. It is assumed that $\Delta P^{\text {exit }}$ is small compared to the other pressure drops in the system. Therefore, only estimates of the pressure drop across PV3 and MV2 are needed. The pressure drop across a valve is difficult to calculate $a$ priori due to the complex dependence of flow on numerous factors. Hence, the flow characteristics of a valve are often empirically determined. As noted earlier, direct measurement of the headspace pressure is not always practical, which complicates the measurement of the pressure drop across any single injection valve. In contrast, characterizing the pressure drop across a bypass valve is straightforward, since a single bypass valve is often the only valve in the flow path in an ampoule bypass configuration. Furthermore, assuming that valves with nominally identical flow coefficients will exhibit a nominally identical dependence of conductance on pressure and flow rate, the bypass valve flow properties can be applied to any other valves with a nominally identical flow coefficient. Such an approach was taken in this work to estimate the pressure drop across PV3 and MV2. For the five-valve cluster ampoules that are the focus of this investigation, the reported valve flow coefficients for all five valves are nominally identical. Therefore, the dependence of valve conductance on pressure and flow rate determined for PV2 (the only single valve for which conductance could be directly characterized) is assumed to be nominally identical to those of the other four valves.

The dependence of valve conductance on pressure and flow rate for PV2 was determined in the following manner. First, $P_{\mathrm{m}}^{\mathrm{CDG} 1}$ and $P_{\mathrm{m}}^{\mathrm{CDG} 2}$ were recorded in the purge configuration at a given carrier gas flow rate at multiple TV settings and for multiple flow rates. Second, $P_{c}^{\text {OUT }}$ was obtained using an equation similar to Eq. (12). Third, for each pair of $P_{\mathrm{m}}^{\mathrm{CDG} 1}$ and $P_{\mathrm{c}}^{\mathrm{OUT}}$ values, the corresponding valve conductance, $C_{\mathrm{v}}\left(\mathrm{m}^{3} / \mathrm{s}\right)$, was calculated using

$$
C_{\mathrm{v}}=\frac{Q}{P_{\mathrm{m}}^{\mathrm{CDG} 1}-P_{\mathrm{c}}^{\mathrm{OUT}}} .
$$

Fourth, the dependence of $C_{\mathrm{v}}$ on $P_{\mathrm{c}}^{\text {OUT }}, C_{\mathrm{v}}\left(P_{\mathrm{c}}^{\text {OUT }}\right)$, at a given flow rate was fit using a quadratic equation. For example, Fig. 4 shows the $C_{\mathrm{v}}$ values (circles) as a function of $P_{\mathrm{c}}^{\mathrm{OUT}}$ at different flow rates and set point temperatures of $75^{\circ} \mathrm{C}, 80{ }^{\circ} \mathrm{C}$, and $90{ }^{\circ} \mathrm{C}$ for the ampoule, valve cluster, and lines, respectively. Also shown are the fits to the data (dashed lines) illustrating that a three-term polynomial is a reasonable function with which to represent $C_{\mathrm{v}}\left(P_{\mathrm{c}}^{\text {OUT }}\right)$. The pressure upstream of a valve can then estimated, assuming that the conductance of each valve in the cluster exhibits the same $C_{\mathrm{v}}\left(P_{\mathrm{c}}^{\text {OUT }}\right)$ function:

$$
P^{\text {up }}=\frac{Q}{C_{\mathrm{v}}\left(P^{\text {down }}\right)}+P^{\text {down }},
$$

where $C_{\mathrm{v}}\left(P^{\text {down }}\right)=C_{\mathrm{v}}\left(P_{\mathrm{c}}^{\text {OUT }}\right)$. Then, the pressure drop across a given valve can be calculated from $\Delta P=P^{\text {up }}-P^{\text {down }}$, for a given flow rate. 


\section{Journal of Research of the National Institute of Standards and Technology}

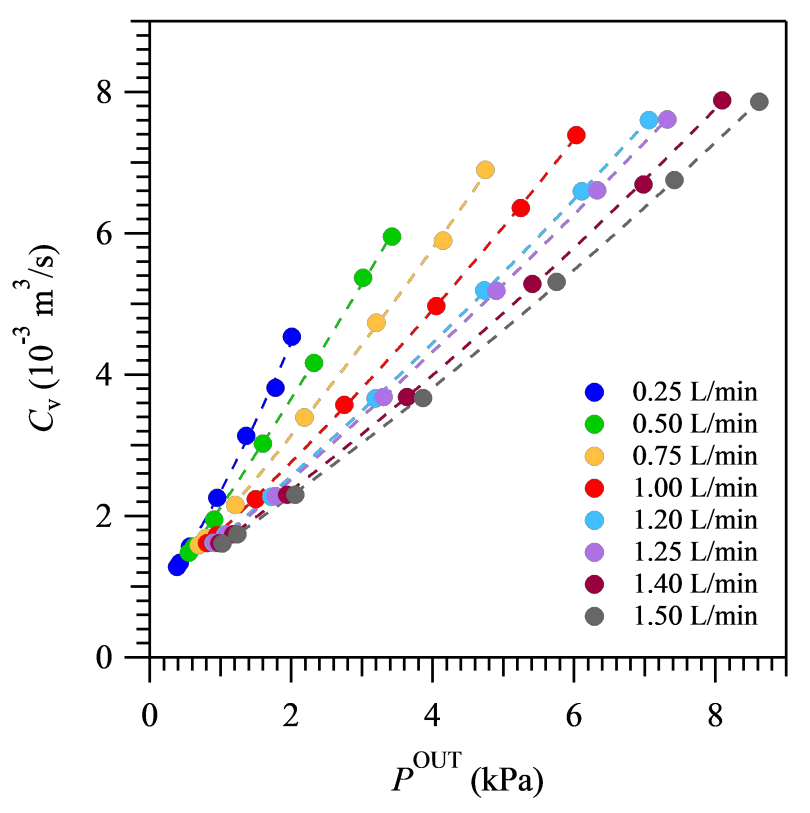

Fig. 4. The $C_{\mathrm{v}}$ values (circles) as a function of $P_{\mathrm{c}}^{\text {Out }}$ and the fits to the data (dashed lines) for different flow rates.

The method for estimating headspace pressure described above was evaluated in the following manner. An additional CDG was temporarily installed on the fill port of an empty bubbler to provide a measurement of the headspace pressure, $P_{\mathrm{m}}^{\mathrm{HS}}$. The three CDG readings were recorded as a function of TV setting and carrier gas flow rate in the injection configuration. For each set of conditions, the pressure was calculated at the ampoule headspace, $P_{\mathrm{c}}^{\mathrm{HS}}$, and $\mathrm{CDG} 1, P_{\mathrm{c}}^{\mathrm{CDG} 1}$, from

$$
P_{\mathrm{c}}^{\mathrm{HS}}=\Delta P^{\mathrm{PV} 3}+\Delta P^{\mathrm{MV} 2}+P_{\mathrm{c}}^{\mathrm{OUT}}
$$

and

$$
P_{\mathrm{c}}^{\mathrm{CDG} 1}=\Delta P^{\mathrm{MV} 1}+\Delta P^{\mathrm{PV} 1}+P_{\mathrm{c}}^{\mathrm{HS}}
$$

The value of $P_{\mathrm{c}}^{\text {OUT }}$ was calculated using Eq. (12), and the pressure drop across each of the valves was calculated from Eq. (15). Figure 5 shows (a) a comparison of the calculated and measured $P^{\mathrm{HS}}$ values and (b) the corresponding relative error for $P_{\mathrm{c}}^{\mathrm{HS}}$. Values are shown for different carrier gas flow rates and set point temperatures of $75^{\circ} \mathrm{C}$, $80^{\circ} \mathrm{C}$, and $90{ }^{\circ} \mathrm{C}$ for the ampoule, valve cluster, and lines, respectively. The relative error varies from $\sim-13 \%$ at low $P_{\mathrm{m}}^{\mathrm{HS}}$ to values between $-2 \%$ and $0 \%$ above $\sim 6 \mathrm{kPa}$. The largest relative error is observed at lower $P_{\mathrm{m}}^{\mathrm{HS}}$ (higher conductance settings) and generally approaches zero with increasing $P_{\mathrm{m}}^{\mathrm{HS}}$. Furthermore, the magnitude of the relative error generally increases with increasing flow rate. In addition, there appears to be a break between the third and fourth data point for each flow rate (at relative errors between $-7 \%$ and $-8 \%$ ), and this break roughly corresponds to two groupings. It is difficult to definitively identify the causes for the observed trends, particularly because gas dynamics in the ampoule are poorly characterized. However, for the lowest pressure data point at $0.25 \mathrm{~L} / \mathrm{min}$ flow rate and the lowest two pressure data points for all other flow rates, flow is choked through PV2 in the bypass configuration. Hence, flow could be choked at the corresponding conditions in the injection 

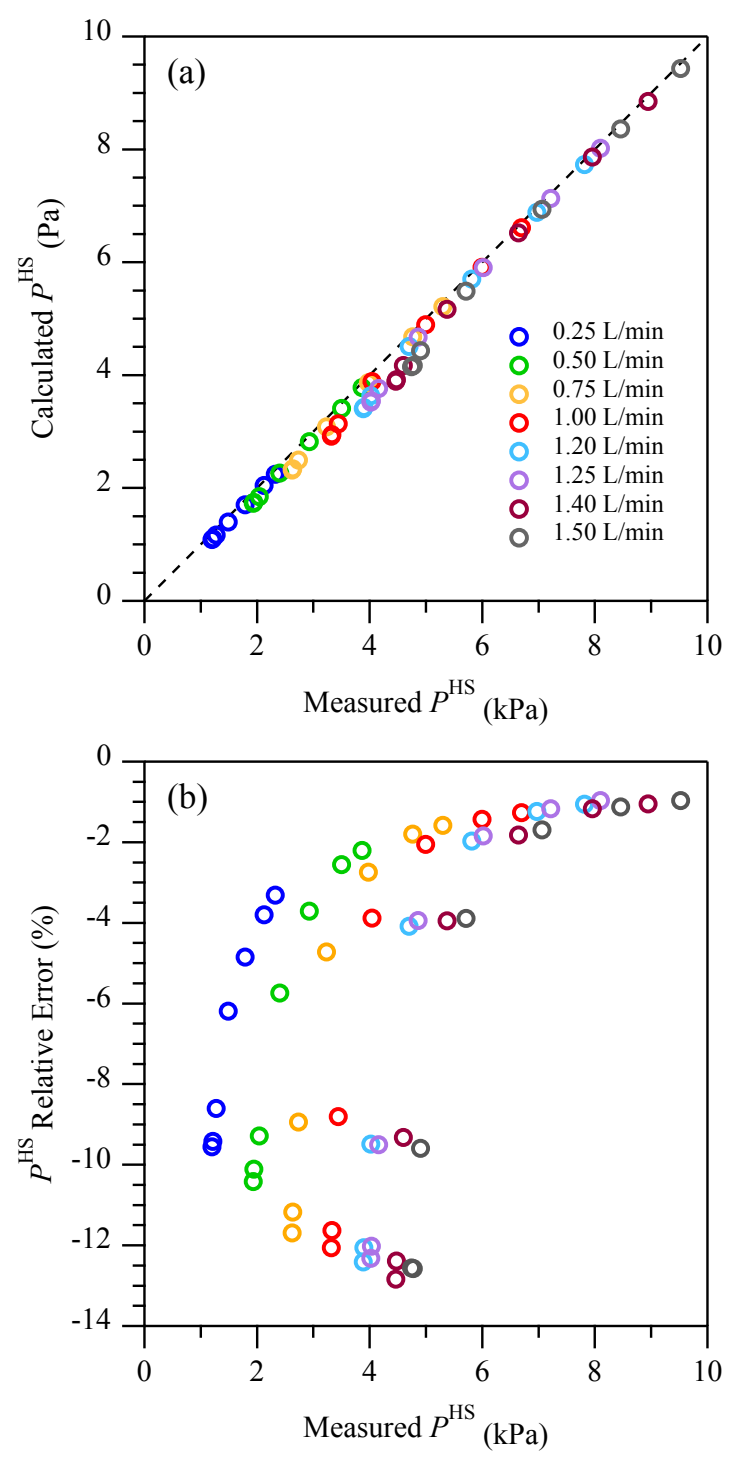

Fig. 5. (a) Calculated and measured $P^{\mathrm{HS}}$ values and (b) corresponding relative error at different carrier gas flow rates.

configuration, which could account for some of the large relative error values, but not all. (In fact, the first two data points at each flow rate also exhibit a weak grouping separate from the third data point.) Gas velocity will increase with increasing flow rate and decreasing pressure. Hence, there is potentially a link between factors leading to higher gas velocity and increased relative error. This method of estimating ampoule headspace pressure is based on empirically characterizing the bypass valve conductance. It is justified if one can neglect (1) $\Delta P^{\text {exit }}$ (which is reasonable if this pressure drop is small relative to other pressure drops in the system) and (2) the conductance of other components in the flow system during injection, e.g., elbows and tubes connecting the valves (which is reasonable if this conductance is large relative to that of the valves). Perhaps this neglect is not justified, at least at the higher flow rates and lower pressures.

Figure 6 shows (a) a comparison of the calculated and measured $P^{\mathrm{CDG} 1}$ values and (b) the corresponding relative error for $P_{\mathrm{c}}^{\mathrm{CDG} 1}$ at the same conditions as Fig. 5. In general, similar trends are observed for $P^{\mathrm{CDG} 1}$ as were for $P^{\mathrm{HS}}$, but the magnitude of the relative errors is somewhat less in the case of $P^{\mathrm{CDG} 1}$. In this case, the relative error varies 


\section{Journal of Research of the National Institute of Standards and Technology}
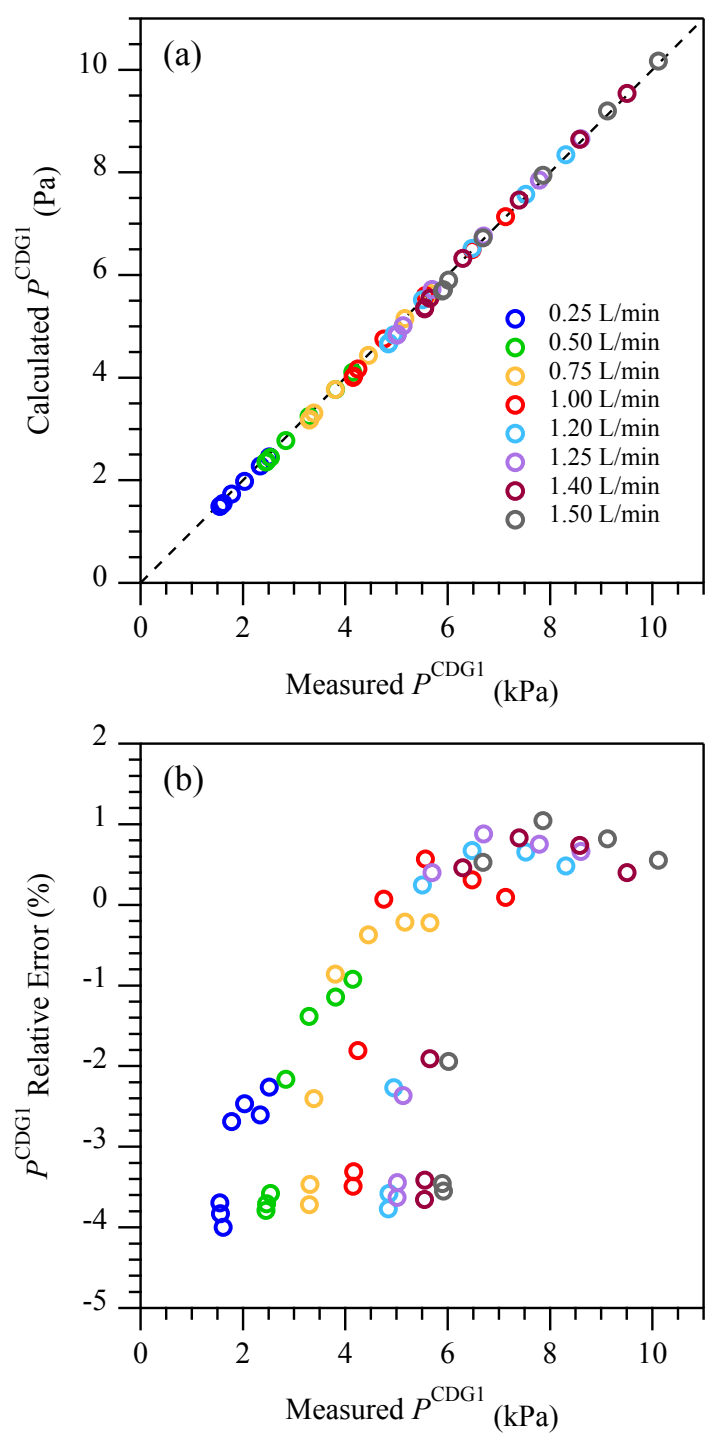

Fig. 6. (a) Calculated and measured $P^{\mathrm{CDG} 1}$ values and (b) corresponding relative error at different carrier gas flow rates.

from $\sim-4 \%$ at low pressures to values between $0 \%$ and $1 \%$ above $\sim 6 \mathrm{kPa}$. The reason for the lower relative errors in the case of $P^{\mathrm{CDG} 1}$ compared to $P^{\mathrm{HS}}$ is not known. Perhaps the reason is related to the neglect of the ampoule entrance and exit pressure drops in this analysis. In this case, some of the effects may be counteracting as flow transitions from the tube into and then out of the ampoule. Whatever the underlying reasons for the errors, it appears that estimates of the headspace pressure within $\sim 10 \%$ of the measured value can be obtained when operating the system under conditions such that choked flow in the valves is avoided. Furthermore, estimates of the headspace pressure within $\sim 2 \%$ of the measured value can be obtained when operating at pressures greater than $\sim 6 \mathrm{kPa}$.

\section{Summary}

The design and performance of an apparatus that, when coupled with an in situ optical technique, can be used to characterize vapor-phase precursor delivery processes was described. The flow system was designed to approximate a single precursor delivery line with an optical flow cell installed downstream of a precursor-containing ampoule. 
Pressure transducers were located upstream of the ampoule and downstream of the flow cell. The downstream pressure can be used to calculate pressure just downstream of the ampoule with relatively small errors, particularly when choked flow through the ampoule valves is avoided. Knowing that line pressure permits determination of the precursor flow rate (when the carrier gas flow rate is known). Subtracting the calculated line pressure from the pressure measured upstream of the ampoule provides an estimation of the maximum pressure drop across the ampoule and ampoule valve cluster. In addition, these transducer readings can be used to estimate the ampoule headspace pressure, in selected situations. Hence, this system can be used to investigate in a general manner the effects on precursor delivery of the delivery apparatus, the ampoule design, and the precursor properties. Such results could facilitate the optimization of deposition recipes and the development of improved ampoule designs.

\section{Acknowledgments}

JEM gratefully acknowledges W. S. Hurst (National Institute of Standards and Technology [NIST]), A. N. Johnson (NIST), W. L. Kimmerle (Nuance Systems Incorporated), and R. K. Kanjolia (EMD Performance Materials) for helpful discussions.

\section{References}

[1] Booker GR, Daly M, Klipstein PC, Lakrimi M, Kuech TF, Li J, Lyapin SG, Mason NJ, Murgatroyd IJ, Portal JC, Nicholas RJ, Symons DM, Vicente P, Walker PJ (1997) Growth of InAs/GaSb strained layer superlattices by MOVPE III. Use of UV absorption to monitor alkyl stability in the reactor. Journal of Crystal Growth 170(1-4):777-782. https://doi.org/10.1016/s0022-0248(96)00578-7

[2] Flynn MG, Smith R, Abraham P, DenBaars S (2001) Control of a III-V MOCVD process using ultraviolet absorption and ultrasonic concentration monitoring. IEEE Transactions on Control Systems Technology 9(5):728-740. https://doi.org/10.1109/87.944468

[3] Gaffney MS, Reaves CM, Smith RS, Holmes AL, DenBaars SP (1996) Control of III-V epitaxy in a metalorganic chemical vapor deposition process: Impact of source flow control on composition and thickness. Journal of Crystal Growth 167(1-2):8-16. https://doi.org/10.1016/0022-0248(96)00204-7

[4] Musolf J (1997) MOCVD with gas phase composition control for the growth of high quality $\mathrm{YBa}_{2} \mathrm{Cu}_{3} \mathrm{O}_{7-x}$ thin films for microwave applications. J Alloys Compd 251(1-2):292-296. https://doi.org/10.1016/s0925-8388(96)02686-2

[5] Roberts JS, Mason NJ, Robinson M (1984) Factors influencing doping control and abrupt metallurgical transitions during atmospheric pressure MOVPE growth of AlGaAs and GaAs. Journal of Crystal Growth 68(1):422-430. https://doi.org/10.1016/00220248(84)90444-5

[6] Stagg JP, Christer J, Thrush EJ, Crawley J (1992) Measurement and control of reagent concentrations in MOCVD reactor using ultrasonics. Journal of Alloys and Compound 120(1-4):98-102. https://doi.org/10.1016/0022-0248(92)90371-o

[7] Thrush EJ, Wale-Evans G, Whiteaway JEA, Lamb BL, Wight DR, Chew NG, Cullis AG, Griffiths RJM (1984) Evidence for transient composition variations at $\mathrm{GaAs} / \mathrm{Ga}_{1-x} \mathrm{Al}_{x} \mathrm{As}$ heterostructure interfaces prepared by metal-organic chemical vapor deposition. Journal of Electronic Materials 13(6):969-988. https://doi.org/10.1007/bf02655311

[8] Thrush EJ, Whiteaway JEA, Wale-Evans G, Wight DR, Cullis AG (1984) Compositional transients in MOCVD grown III-V heterostructures. Journal of Crystal Growth 68(1):412-421. https://doi.org/10.1016/0022-0248(84)90443-3

[9] Zemon S, Black J, Norris P, Lee J, Lambert G (1984) Characterization of LP-MOCVD grown (Al, Ga)As/GaAs heterostructures by photoluminescence: Single heterojunction and inadvertent quantum wells. Japanese Journal of Applied Physics Part 2-Letters 23(12):L925-L927. https://doi.org/10.1143/jjap.23.1925

[10] Agahi F, Lutz CR, Lau KM (1994) Improvement of gas-switching abruptness for atmospheric-pressure organometallic vapor-phase epitaxy. Journal of Crystal Growth 139(3-4):344-350. https://doi.org/10.1016/0022-0248(94)90186-4

[11] Sillmon RS, Bottka N, Butler JE, Gaskill DK (1986) An ultra-fast gas delivery system for producing abrupt compositional switching in OMVPE. Journal of Crystal Growth 77(1-3):73-78. https://doi.org/10.1016/0022-0248(86)90284-8

[12] Holstein WL (1994) Performance of gas saturators in the presence of exit stream temperature gradients and implications for chemical vapor deposition saturator design. Chemical Engineering Science 49(13):2097-2105. https://doi.org/10.1016/0009-2509(94)E0039-S

[13] Shenai-Khatkhate DV, DiCarlo RL, Marsman CJ, Polcari RF, Ware RA, Woelk E (2007) Stable vapor transportation of solid sources in MOVPE of III-V compound semiconductors. Journal of Crystal Growth 298:176-180. https://doi.org/10.1016/j.jcrysgro.2006.10.195

[14] Shenai-Khatkhate DV, Ware RA, DiCarlo RL, Polcari RF, Marsman CJ, Woelk E, Keiter AG (2006) Exceptionally stable vapor delivery of trimethylindium under intense OMVPE growth conditions. Journal of Crystal Growth 287(2):679-683. https://doi.org/10.1016/j.jcrysgro.2005.10.095

[15] Timmons M, Rangarajan P, Stennick R (2000) A study of cylinder design for solid OMVPE sources. Journal of Crystal Growth 221:635-639. https://doi.org/10.1016/s0022-0248(00)00791-0

[16] Love A, Middleman S, Hochberg AK (1993) The dynamics of bubblers as vapor delivery systems. Journal of Crystal Growth 129(12):119-133. https://doi.org/10.1016/0022-0248(93)90441-x 
[17] Woelk E, DiCarlo R (2014) Control of vapor feed from liquid precursors to the OMVPE process. Journal of Crystal Growth 393:3234. https://doi.org/10.1016/j.jcrysgro.2013.10.020

[18] Knauf J, Schmitz D, Strauch G, Jurgensen H, Heyen M, Melas A (1988) Comparison of ethyldimethylindium (EDMIn) and trimethylindium (TMIn) for GaInAs and InP growth by LP-MOVPE. Journal of Crystal Growth 93(1-4):34-40. https://doi.org/10.1016/0022-0248(88)90502-7

[19] Hodgkinson J, Tatam RP (2013) Optical gas sensing: A review. Measurement Science \& Technology 24(1):012004. https://doi.org/10.1088/0957-0233/24/1/012004

[20] Herman IP (1996) Section 1.5 Survey of Optical Probes. Optical Diagnostics for Thin Film Processing (Academic Press, Inc., San Diego, CA), pp 21-28.

[21] Betsch RJ (1986) Parametric analysis of control parameters in MOCVD. Journal of Crystal Growth 77(1-3):210-218. https://doi.org/10.1016/0022-0248(86)90303-9

[22] Hersee SD, Ballingall JM (1990) The operation of metalorganic bubblers at reduced pressure. Journal of Vacuum Science \& Technology, A: Vacuum, Surfaces, and Films 8(2):800-804. https://doi.org/10.1116/1.576921

[23] Middleman S (1991) Some problems related to the performance of an evaporator as a vapor delivery system. Journal of Crystal Growth 114(1-2):13-21. https://doi.org/10.1016/0022-0248(91)90672-r

[24] Middleman S, Hochberg AK (1993) Section 10-4.5. Doping from a Liquid Source—Dynamics of a Bubbler. Process Engineering Analysis in Semiconductor Device Fabrication (McGraw-Hill, New York, NY), pp 356-360.

[25] Fau-Canillac F, Maury F (1994) Control of the uniformity of thickness of Ni thin films deposited by low pressure chemical vapor deposition. Surface \& Coatings Technology 64(1):21-27. https://doi.org/10.1016/S0257-8972(09)90081-0

[26] Mayer B, Collins CC, Walton M (2001) Transient analysis of carrier gas saturation in liquid source vapor generators. Journal of Vacuum Science \& Technology, A: Vacuum, Surfaces, and Films 19(1):329-344. https://doi.org/10.1116/1.1322646

[27] Maury F, Duminica FD, Senocq F (2007) Optimization of the vaporization of liquid and solid CVD precursors: Experimental and modeling approaches. Chemical Vapor Deposition 13(11):638-643. https://doi.org/10.1002/cvde.200706600

[28] Blomberg T (2013) Unit Steps of an ALD Half-Cycle. ECS Transactions 58(10):3-18. https://doi.org/10.1149/05810.0003ecst

[29] Sullivan JJ, Schaffer S, Jacobs RP (1989) Mass flow measurement and control of low vapor pressure sources. Journal of Vacuum Science \& Technology, A: Vacuum, Surfaces, and Films 7(3):2387-2392. https://doi.org/10.1116/1.575905

[30] Woelk E (2010) Performance of a central delivery system for metalorganic precursors. Journal of Crystal Growth 312(8):1340-1342. https://doi.org/10.1016/j.jcrysgro.2009.09.045

[31] Maslar JE, Kimes WA, Sperling BA, Kanjolia RK (2017) Nondispersive infrared gas analyzer for vapor density measurements of a carbonyl-containing organometallic cobalt precursor. Applied Spectroscopy 71(12):2632-2642. https://doi.org/10.1177/0003702817716939

[32] Kimes WA, Moore EF, Maslar JE (2012) Perpendicular-flow, single-wafer atomic layer deposition reactor chamber design for use with in situ diagnostics. Review of Scientific Instruments 83(8):083106-083101. https://doi.org/10.1063/1.4742991

[33] O'Hanlon JF (1989) Gas Flow. A User's Guide To Vacuum Technology (John Wiley \& Sons, Inc, New York, NY, USA), Chapter 3 , 2nd Ed., pp 25-53.

[34] National Institute of Standards and Technology (2016) NIST Standard Reference Database 23 (REFPROP), version 9.0. Available at https://www.nist.gov/srd/refprop

About the authors: James E. Maslar is a research chemist in the Chemical Sciences Division at NIST. His present research focuses on the development of in situ metrologies for characterizing and controlling chemical precursor delivery for vapor deposition processes.

William A. Kimes is a research physicist in the Chemical Sciences Division at NIST. His present research focuses on the development and maintenance of gas standard reference materials.

Brent A. Sperling is a research chemist in the Chemical Sciences Division at NIST. His present research focuses on in situ optical and infrared measurements during materials processing.

The National Institute of Standards and Technology is an agency of the U.S. Department of Commerce. 